\title{
Effects of vitamin $C$ on oxidative status in pigs anesthetized with isoflurane
}

\author{
Jae Yeon Lee, Myung Cheol Kim* \\ College of Veterinary Medicine, Chungnam National University, Daejeon 305-764, Korea
}

(Received: April 27, 2012; Revised: June 17, 2012; Accepted: June 20, 2012)

\begin{abstract}
This study investigated the effects of vitamin $\mathrm{C}$ on oxidative stress induced by volatile anesthetics in pigs. One group of pigs was used as an anesthesia control group (group 1), and they were anesthetized with isoflurane in oxygen and saline $(0.9 \% \mathrm{NaCl})$ was injected intravenously. The other group (group 2) was anesthetized with isoflurane and injected intravenously with vitamin $\mathrm{C}$. Total oxidant status, total antioxidant status, and the oxidative stress index in group 2 were significantly different compared with those in group 1 . The results showed that intravenous administration of vitamin $\mathrm{C}$ decreased oxidative stress during isoflurane anesthesia in pigs.
\end{abstract}

Keywords : anesthesia, isoflurane, oxidative stress, pigs, vitamin C

\section{Introduction}

Reactive oxygen species (ROS) are generated in excess due to damage to cell structures, and these accumulate leading to a condition known as oxidative stress. Volatile anesthesia induces ischemia-reperfusion injury and is a complex phenomenon resulting in ROS production [1]. Previous studies in animals have suggested that exposure to volatile anesthetics can induce oxidative stress [5, 6]. Isoflurane is a less hepatotoxic inhalation anesthetic than others, as it undergoes quantitatively much less metabolism from toxic reactive intermediates [4]. However, isoflurane is cytotoxic in vitro [3], whereas the oxidative stress issue is still controversial. The possibility of potential side effects from inhalation anesthetics has been the subject of various studies. Nevertheless, the oxidant and antioxidant status of isoflurane in animals has not been fully evaluated.

Several antioxidant supplements increase antioxidant activity. Vitamin C, for one, may protect cellular membrane lipoproteins against oxidative damage caused by toxic free radicals at the early stage [7]. These antioxidant functions of vitamin $C$ may prevent certain types of oxidative stress due to anesthetics. Therefore, we investigated the protective effects, if any, of intravenously injected vitamin $\mathrm{C}$ on oxidant and antioxidant status in the plasma of pigs anesthetized with isoflurane.

\section{Materials and Methods}

\section{Animals}

Eighteen male Landrace and Yorkshire mixed pigs (37.6 \pm
$1.7 \mathrm{~kg}, 3 \sim 4$ month old) were used. Pigs were randomly assigned to a control untreated group (group 1, n =9) or an ascorbic acid-treated group (group 2, $n=9$ ). All pigs were obtained from the experimental livestock farm of the College of Agriculture, Chungnam National University (CNU), Korea. These experimental and housing protocols were approved by the CNU Animal Care and Use Committee (approval no. 2010-2-10). Pigs were starved for $12 \mathrm{~h}$ prior to surgery in order to prevent any possible adverse effects associated with anesthesia.

\section{Anesthesia and drug administrations}

Anesthesia was induced and maintained with $2 \%$ delivered isoflurane carried in oxygen using a face mask. The ascorbic acid treatment (Ascorbic Acid Injection; Huons, Korea; $300 \mathrm{mg}$, IV) was administered prior to anesthesia induction. The control pigs were received intravenously saline $(0.9 \% \mathrm{NaCl})$ alone. A gas mixture $(100 \%$ oxygen and $2 \%$ isoflurane) was administered for $1 \mathrm{~h}$. Pigs were attached to a standard monitoring device (Pulscan-Component, Patient monitor; Scionic, Korea). The heart rate (HR), respiratory rate (RR), oxygen saturation $\left(\mathrm{SpO}_{2}\right)$ and rectal temperature (RT) were measured by using a patient monitor (PulscanComponent, Patient monitor; Scionic) and were recorded.

\section{Obtaining of blood and plasma}

Venous blood samples were collected into heparin tubes via venipuncture from the jugular vein at designated times. Plasma was separated by centrifugation $(1,000 \times \mathrm{g}$ for 10 $\min$ ) and stored at $-80^{\circ} \mathrm{C}$. Plasma total oxidant status (TOS) and total antioxidant status (TAS) levels were determined to

*Corresponding author

Tel: +82-42-821-6757, Fax: +82-42-821-8903

E-mail: mckim@cnu.ac.kr 
Table 1. Oxidative stress parameters of the pigs

\begin{tabular}{ccccc}
\hline \hline & Group & Before induction of anesthesia & End of the anesthesia & $1 \mathrm{~h}$ after the end of anesthesia \\
\hline TOS & Group 1 & $217.36(11.6)$ & $276.03(21.09)^{*}$ & $240.09(25.7)$ \\
$\left(\mu \mathrm{molH}_{2} \mathrm{O}_{2} / \mathrm{L}\right)$ & Group 2 & $214.47(12.26)$ & $192.29(30.36)^{* * *}$ & $191.0(27.01)^{* *}$ \\
TAS & Group 1 & $27.1(2.08)$ & $20.5(1.45)^{*}$ & $22.4(2.94)^{*}$ \\
$(\mathrm{mmol} \mathrm{Trolox} \mathrm{equiv/L)}$ & Group 2 & $27.1(1.90)$ & $30.1(9.33)^{* *}$ & $29.4(8.15)^{* *}$ \\
OSI & Group 1 & $8.0(0.95)$ & $13.5(1.87)^{*}$ & $10.9(1.86)^{*}$ \\
$($ arbitrary unit) & Group 2 & $7.9(0.57)$ & $6.7(2.58)^{* *}$ & $6.8(1.91)^{* *}$ \\
\hline
\end{tabular}

The values represent the median (inter-quartile range). "Statistically difference compared to "before induction of anesthesia" $(n=9)$.

${ }^{* *}$ Statistically difference compared to group $1(n=9)$.

evaluate oxidative stress using a commercially available kit developed by Erel [2] (Mega Tip, REL Assay Diagnostics; Gaziantep, Turkey). The ratio of TOS to TAS was the oxidative stress index (OSI), an indicator of the degree of oxidative stress.

\section{Statistical analysis}

Statistical analysis was performed using SPSS software for Windows, release 18.0 (SPSS, USA). Results are expressed as median (inter-quartile range). A Mann-Whitney $U$-test was applied with a $p<0.05$ being considered significant.

\section{Results}

Plasma TOS, TAS, and OSI values are shown in Table 1. Decreases in plasma TOS and OSI levels in group 2 after anesthesia were observed, whereas increases in group 1 were observed. The group 1 TOS level was significantly higher at the end of anesthesia than that before the induction of anesthesia $(p=0.016)$. Plasma TAS levels increased in group 2 after anesthesia, whereas they decreased in group 1. The TAS level of group 1 was significantly lower at the end of anesthesia and $1 \mathrm{~h}$ after the end of anesthesia than before induction of anesthesia ( $p=0.04$ and 0.01 , respectively). The levels of TOS, TAS and OSI in group 2 were significantly different compared with those of group 1 at the end of anesthesia ( $p=0.01,0.006$, and 0.006 , respectively) and $1 \mathrm{~h}$ after the end of anesthesia $(p=0.016,0.037$, and 0.006 , respectively).

\section{Discussion}

The results showed that an intravenous administration of ascorbic acid decreased oxidative damage during isoflurane anesthesia in pigs. Isoflurane anesthesia significantly increased the TOS and OSI values and decreased the TAS values in the control group. In contrast, decreases in TOS and OSI levels and increases in TAS levels were observed in the ascorbic acid treated group.

Free radical species are constantly produced as a normal consequence of aerobic metabolism. Oxidative stress results from an imbalance between radical-generating and radical scavenging system, leading to cell membrane impairment or DNA damage. Hydrogen peroxide and other derivatives of peroxides, produced physiologically in organisms and occurring in higher concentrations under some pathologic conditions, diffuse into plasma [2].The level of total peroxide was measured and expressed as TOS in this study. The percentage of ratio of the TOS to the TAS gave the OSI, an indicator of the degree of oxidative stress. The evaluation of oxidative stress can indirectly reflect the changes in microcirculation in the organs during anesthesia.

Many oxidative stress markers, including nitric oxide, lipid peroxidation, protein carbonyl content, and protein sulfhydryl have been investigated. In addition to these stress parameters, plasma TOS and TAS levels have been used to reflect oxidative stress and actions against oxidative stress, respectively. An evaluation of these oxidative stress parameters can indirectly reflect changes in organ microcirculation during anesthesia [2]. The blood contains many antioxidant molecules that prevent and inhibit the harmful effects of ROS. The effects of total antioxidant species reflect the antioxidative status of plasma. The cooperative actions of these various antioxidants in plasma protect organs against oxidative stress. In spite of the fact, blood vitamin C concentrations were not determined in this study, but increases in TAS levels were thought to be related to the ascorbic acid treatment. As a consequence, the results showed that vitamin $\mathrm{C}$ is capable of increasing the antioxidant capacity of plasma in pigs.

In conclusion, we observed that isoflurane increased oxidative stress parameters. In the light of present results, vitamin $\mathrm{C}$ may play a role in the prevention of oxidative injury produced by volatile anesthesia. However, more detailed studies are needed to assess the possible relationships between antioxidants and isoflurane.

\section{Acknowledgments}

This work was supported by the Basic Science Research Program through the National Research Foundation of Korea (NRF) funded by the Ministry of Education, Science and Technology (No.2010-0024553). 


\section{References}

1. Allaouchiche B, Debon R, Goudable J, Chassard D, Duflo F. Oxidative stress status during exposure to propofol, sevoflurane and desflurane. Anesth Analg 2001, 93, 981-985.

2. Erel O. A novel automated direct measurement method for total antioxidant capacity using a new generation, more stable ABTS radical cation. Clin Biochem 2004, 37, 277285.

3. Jaloszyński P, Kujawski M, Wasowicz M, Szulc R, Szyfter K. Genotoxicity of inhalation anesthetics halothane and isoflurane in human lymphocytes studied in vitro using the comet assay. Mutat Res 1999, 439, 199-206.
4. Kenna JG, Jones RM. The organ toxicity of inhaled anesthetics. Anesth Analg 1995, 81 (6 Suppl), S51-66.

5. Naziroğlu M, Cay M. Protective role of intraperitoneally administered vitamin $\mathrm{E}$ and selenium on the antioxidative defense mechanisms in rats with diabetes induced by streptozotocin. Biol Trace Elem Res 2001, 79, 149-159.

6. Naziroğlu M, Günay C. The levels of some antioxidant vitamins, glutathione peroxidase and lipoperoxidase during the anaesthesia of dogs. Cell Biochem Funct 1999, 17, 207-212.

7. Netke SP, Roomi MW, Tsao C, Niedzwiecki A. Ascorbic acid protects guinea pigs from acute aflatoxin toxicity. Toxicol Appl Pharmacol 1997, 143, 429-435. 